Sky Polarisation at Far-Infrared to Radio Wavelengths

F. Boulanger, M.-A. Miville-Deschênes (eds)

EAS Publications Series, Vol. ?, 2021

\title{
POLARIZED EMISSION FROM INTERSTELLAR DUST
}

\author{
John E. Vaillancourt ${ }^{1}$
}

\begin{abstract}
Observations of far-infrared (FIR) and submillimeter (SMM) polarized emission are used to study magnetic fields and dust grains in dense regions of the interstellar medium (ISM). These observations place constraints on models of molecular clouds, star-formation, grain alignment mechanisms, and grain size, shape, and composition. The FIR/SMM polarization is strongly dependent on wavelength. We have attributed this wavelength dependence to sampling different grain populations at different temperatures. To date, most observations of polarized emission have been in the densest regions of the ISM. Extending these observations to regions of the diffuse ISM, and to microwave frequencies, will provide additional tests of grain and alignment models.

An understanding of polarized microwave emission from dust is key to an accurate measurement of the polarization of the cosmic microwave background. The microwave polarization spectrum will put limits on the contributions to polarized emission from spinning dust and vibrating magnetic dust.
\end{abstract}

\section{Introduction}

The dominant source of Galactic emission at far-infrared (FIR) and submillimeter $(\mathrm{SMM})$ wavelengths $(\lambda \sim 50 \mu \mathrm{m}-1 \mathrm{~mm}, \nu \sim 300-6000 \mathrm{GHz})$ is thermal emission from interstellar dust at temperatures of $10-100 \mathrm{~K}$. In dense regions of the interstellar medium (ISM) such as molecular clouds, this emission has measurable polarization at almost every point (e.g. Dotson et al. 2000, 2006). This polarization is due to the alignment of the dust grains with interstellar magnetic fields (Davis \& Greenstein 1951). As a result of the magnetic alignment mechanism, polarimetry allows detailed studies of magnetic fields (e.g. Chuss et al. 2003; Matthews et al. 2001; Crutcher 2006).

Polarimetry can also be used to place constraints on grain properties, cloud environments, and grain alignment efficiency (Hildebrand et al. 1999; Whittet et al.

\footnotetext{
${ }^{1}$ Enrico Fermi Institute, University of Chicago, Chicago, Illinois, USA

e-mail: johnv@oddjob.uchicago.edu
} 
2001; Lazarian. Goodman. \& Mvers 1997). Observations at multiple wavelengths are especially useful for such studies. At optical and near-infrared wavelengths, spectropolarimetry puts constraints on grain size, shape, and composition Aitken 1996; Whittet 2004; Martin 2006). In molecular clouds, the FIR/SMM polarization spectrum is observed to fall from $60 \mu \mathrm{m}$ to $350 \mu \mathrm{m}$ and rise again to $1300 \mu \mathrm{m}$. We attribute this spectrum to correlations between temperature and alignment efficiency (Hildebrand et al. 1999; Vaillancourt 2002).

Further tests of grain and alignment models require that measurements of the polarization spectrum be extended to more diffuse regions of the ISM and to longer wavelengths. Diffuse infrared cirrus clouds present simpler physical environments than dense star-forming molecular clouds. Long wavelength observations $(\lambda \geqslant 850 \mu \mathrm{m})$ will determine the behavior of the polarization spectrum where thermal emission from dust no longer dominates the total spectral energy distribution. Experiments such as Planck (Tauber 2004) and the Wilkinson Microwave Anisotropy Probe (WMAP; Hinshaw et al. 2003) are designed to make all-sky polarization measurements at these wavelengths.

Dust may also be a major contributor to the observed Galactic emission at microwave frequencies $(\lambda \sim 3-300 \mathrm{~mm})$. This dust emission will be dominated by electric dipole (Draine \& Lazarian 1998b) or magnetic dipole (Draine \& Lazarian 1999) radiation rather than thermal radiation. As a result, Galactic dust emission may be an important foreground contributor to studies of the cosmic microwave background (CMB). Extension of the polarization spectrum of Galactic clouds from the SMM to the microwave will be key to accurately removing this polarized foreground from the CMB.

\section{Polarization by Absorption and Emission}

\subsection{Polarization by Absorption}

Polarization of starlight at ultraviolet (UV), visible (Vis), and near-infrared (IR) wavelengths is due to selective extinction by aspherical grains which have been aligned by a local magnetic field (Davis \& Greenstein 1951). Grain alignment generally occurs in two steps: 1) the grain axis with the largest moment of inertia becomes aligned with the spin (angular momentum) axis and 2) the spin axis then becomes aligned with the local magnetic field (e.g. Lazarian 2003; Whittet 2003; Roberge 2004; Lazarian \& Cho 2005). While grain dynamics are certainly more complex than this picture (Lazarian \& Yan 2004), and other alignment mechanisms (e.g. mechanical alignment; Gold 1952) may be important in some regions (Hildebrand 1988; Lazarian 2003), this general alignment picture will suffice for the discussion of polarimetry presented here.

Since starlight is preferentially absorbed along the long axis of the grain, the net polarization will be parallel to the magnetic field. Observations of starlight polarization by absorption have proven to be a valuable tool for tracing the magnetic field structure in diffuse regions of the interstellar medium (e.g. Heiles 2000; Berdvugin. Piirola. \& Teerikorpi 2004; and references therein). However, at high 

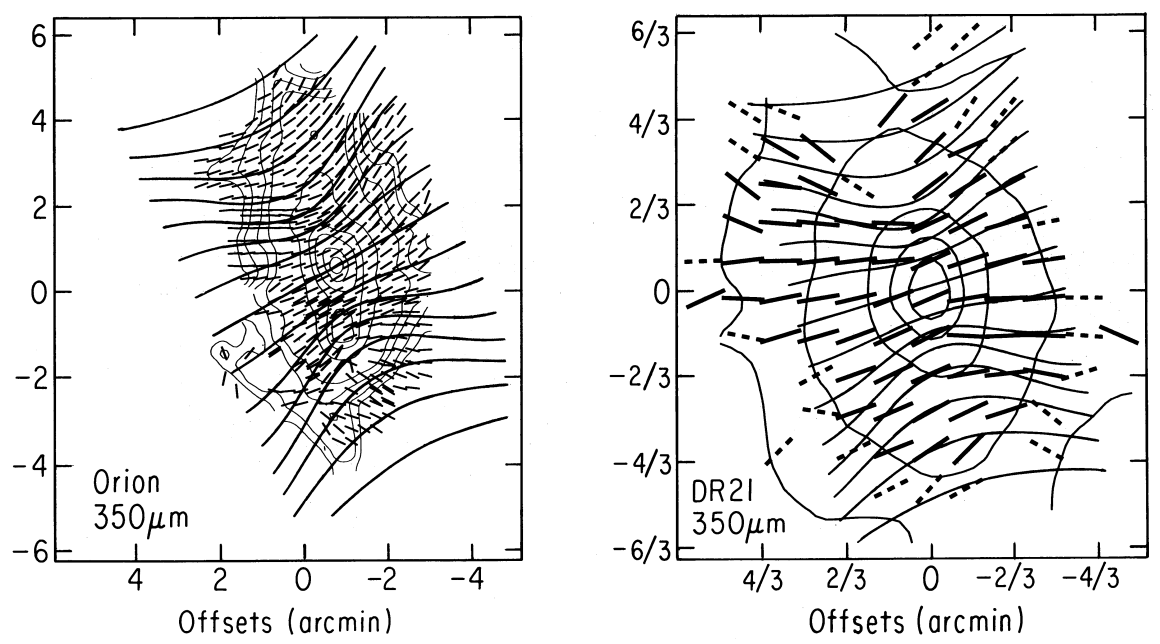

Fig. 1. Polarization of the Orion Molecular Cloud (left; Houde et al. 2004) and DR21 (right; Dotson et al. 2006) at $350 \mu \mathrm{m}$. Polarization vectors (small solid and dashed lines) are all drawn the same size and are rotated 90 degrees to show the inferred magnetic field direction. The curves show a possible model for the magnetic field. For DR21 the curves are scaled down by a factor of 3 to account for the relative distances of the two clouds.

extinctions, UV/Vis/IR light is completely absorbed. Even at moderate extinctions $\left(A_{V} \gtrsim 1\right.$ magnitude) polarization by absorption is not a reliable tracer of the magnetic field due to the drop in grain alignment efficiency (3.2 Goodman et al. 1995; Arce et al. 1998; Bastien 2006).

\subsection{Polarization by Emission}

The net polarization of radiation emitted from dust grains is parallel to the long axis of the grain, perpendicular to the aligning magnetic field. Figure 1 shows an example of magnetic fields in two molecular clouds inferred from emission polarimetry at SMM wavelengths.

Instrument sensitivity, as well as atmospheric absorption and stability, set a lower limit on the column densities which can be traced using emission polarimetry. At present the best submillimeter instruments require $A_{V} \gtrsim 10-20$ (Novak et al. 2004). As a result polarimetry using emission and absorption trace magnetic fields in different domains of the ISM. Figure 2 shows that the Galactic magnetic field can be quite different in these regions. Optical polarimetry traces a field which is in general parallel to the Galactic plane, whereas the FIR polarization vectors appear to be oriented randomly. While polarimetry in dense clouds clearly traces magnetic fields (3.4) they do not exhibit a clear link to the large-scale Galactic 

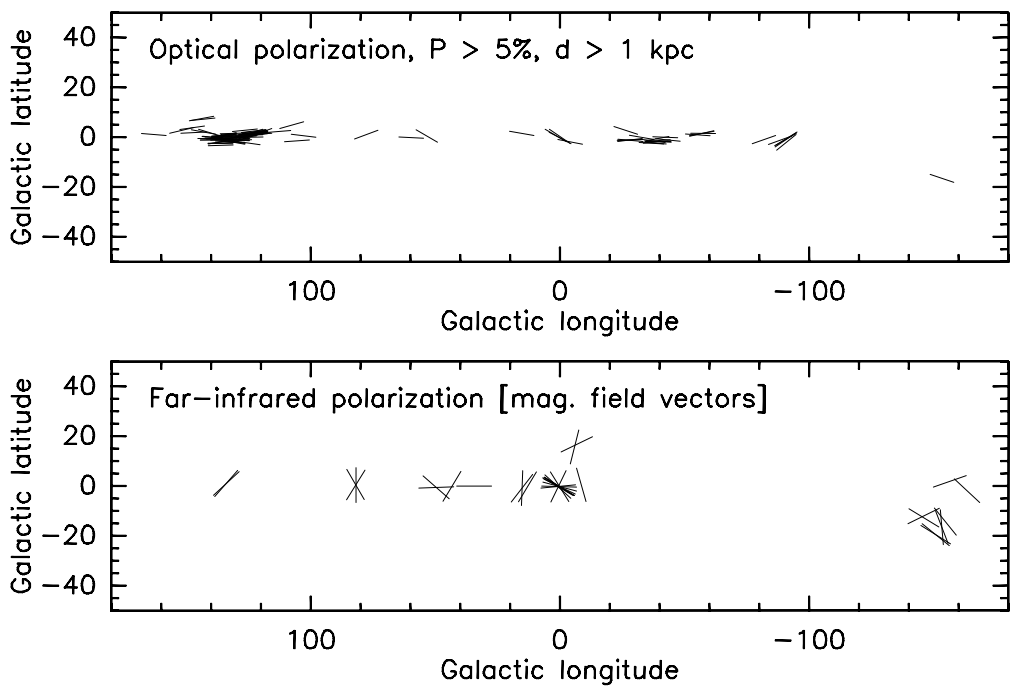

Fig. 2. Top: Galactic polarization measurements of the diffuse ISM at optical wavelengths (Heiles 2000). Bottom: Mean field direction for 27 Galactic molecular clouds observed in the far-infrared and submillimeter (Hildebrand 2001; courtesy C. D. Dowell).

field.

\section{Grain Alignment}

\subsection{Radiative Torques}

The alignment of the rotational and symmetry axes of dust grains with magnetic fields is opposed by collisions between the grains and gas molecules. In order for the grains to become aligned, the timescale for alignment must be faster than the timescale of the collisional damping. Purcell (1979) showed that this condition is satisfied when the grains are rotating suprathermally, $E_{\text {rot }} \gg k T$. He showed further that torques produced by the formation and subsequent ejection of $\mathrm{H}_{2}$ molecules from grain surfaces could spin-up grains to the necessary speeds.

The necessary torques can also be applied by photons (Dolginov 1972; Dolginov \& Mvtrophanov 1976; Draine \& Weingartner 1996, 1997). Photons will produce a net torque on irregularly shaped grains because they present different cross-sections to rightand left-circularly polarized photons. Several observations provide support for the existence of these radiative torques (Lazarian 2003). Some of these are discussed further in the remainder of $\$ 3$ and in 4

Radiative torques are theoretically more attractive than $\mathrm{H}_{2}$ torques due to "thermal flipping" and "thermal trapping" of grains. Lazarian \& Draine (1997, 1999b) showed that thermal fluctuations in a grain may result in sudden flips of 
its angular momentum vector. Since the location of $\mathrm{H}_{2}$ formation is dependent on grain surface structure the resulting torques will change direction when the spin vector flips, causing the grain to spin-down. Grains smaller than $1 \mu \mathrm{m}$ cannot then reach suprathermal velocities (Lazarian \& Draine 1999a). However, this is not the case for radiative torques because a grain's helicity is not changed by a change in the grain's orientation.

\subsection{Limits on Polarization by Absorption}

If all grains are aligned with equal efficiency regardless of their environment then the number of aligned grains increases at the same rate as the total number of grains. One then expects measurements of polarization by absorption to increase towards regions of higher dust column density. Arce et al. (1998) have shown that this expectation holds only for low extinctions. At low extinctions interstellar photons can penetrate the cloud and spin-up the dust grains via radiative torques but at higher extinctions the cloud is opaque to UV/Vis/IR photons. Also, as the extinction increases the polarization is further reduced by scattered photons. As a result, Arce et al. (1998) conclude that polarization by absorption cannnot be used to reliably trace magnetic field structure in regions where $A_{V} \gtrsim 1.3$

If radiative torques are required to spin-up grains to the angular velocities necessary for alignment then two questions must be answered in regard to dense molecular clouds where extinctions can be much larger than $A_{V} \approx 1.3$. What is the mechanism for producing grain alignment in these clouds? Does the FIR/SMM polarization reliably trace the orientation of magnetic fields?

\subsection{Radiative Torques in Dense Clouds}

Even in dense clouds, radiative torques can spin grains up to the suprathermal velocities required for alignment. While photons from the interstellar radiation field (ISRF) cannot penetrate deeply into the cloud interior (where $A_{V} \gg 1.3$ ), photons from embedded stars can provide the necessary torques. Cho \& Lazarian (2005) have shown that radiative torques are still efficient up to $A_{V} \sim 10$. Since clouds are likely inhomogeneous, radiation can leak out to regions of even higher density. In addition, the efficiency of radiative torques rises sharply with increasing grain size (Cho \& Lazarian 2005). Due to the increased efficiency of grain growth mechanisms (coagulation, mantle growth) grains tend to be larger in dense clouds than in the diffuse ISM (Whittet 2003).

Stellar photons will also heat the grains, resulting in a correlation between grain temperature and alignment efficiency which is observable (4 Hildebrand et al. 1999). This correlation will be strongest along lines of sight to embedded stars and has been observed towards the H II regions W3A (Schleuning et al. 2000) and M42 (Vaillancourt 2002). 


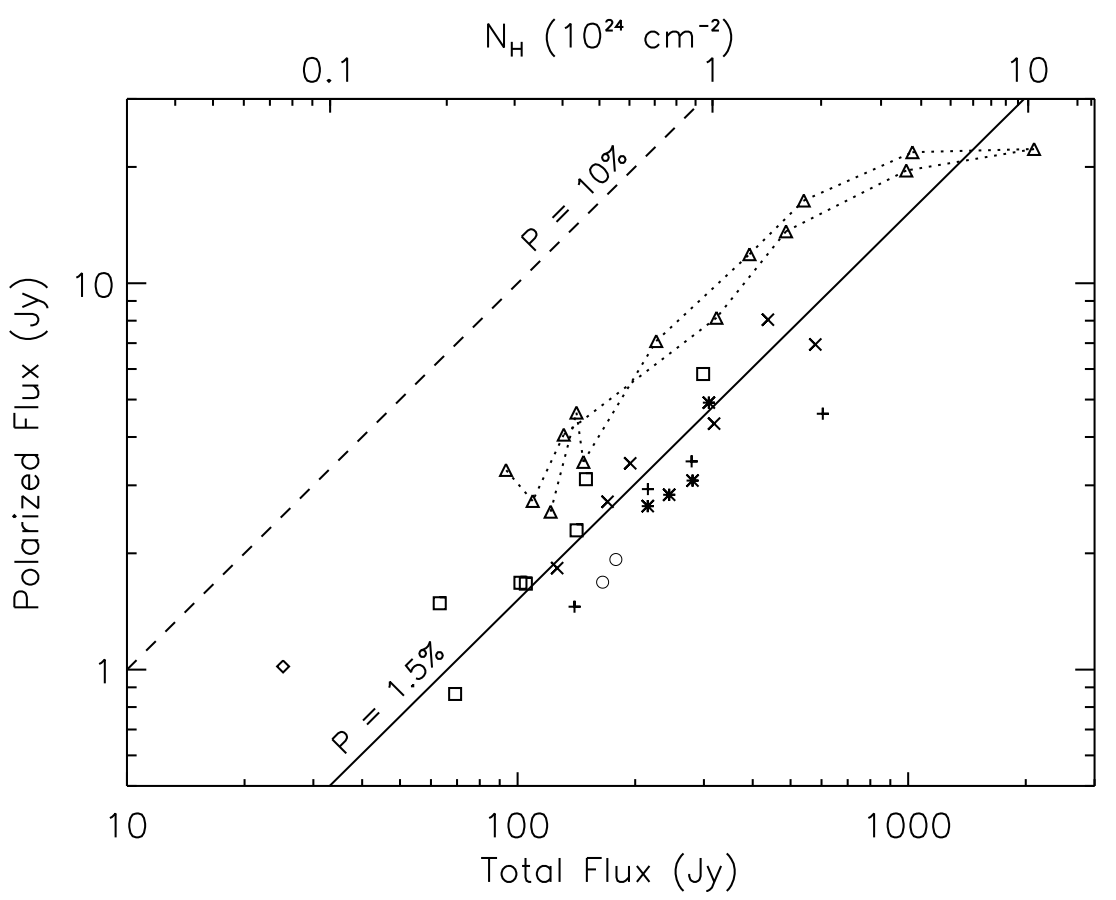

Fig. 3. Polarized flux vs. total flux at $350 \mu \mathrm{m}$ for selected points in six molecular clouds (Vaillancourt et al. 2006, in preparation). Data points (from Dotson et al. 2006) have $P>3 \sigma_{p}$ and were chosen to minimize changes in polarization position angle (and therefore changes in the projected magnetic field direction) between spatially adjacent points. The solid line is the best linear fit to a constant polarization $(P=1.5 \%)$ and the dashed line is drawn at a constant polarization of $10 \%$. A dotted line connects data at a constant declination through the core of the Orion Molecular Cloud (Figure 1); note the turn over at high densities suggesting a loss in grain alignment. Fluxes are measured with a $20^{\prime \prime}$ beam. The scale at the top is the hydrogen column density for a dust temperature of $35 \mathrm{~K} ; N_{\mathrm{H}}=9.2 \times 10^{24} \mathrm{~cm}^{-2}$ for $\tau(350 \mu \mathrm{m})=1$ (Hildebrand 1983).

\subsection{Polarized Submillimeter Flux}

The assumption that emission polarimetry traces magnetic fields can be tested. Again, if all grains are aligned with equal efficiency then one expects the product of the polarization by emission and column density to scale with column density. Figure 3 plots this product in a selection of six molecular clouds using the $350 \mu \mathrm{m}$ flux as a tracer of column density. The results are consistent with the expectations of efficient grain alignment. This trend becomes shallower for some of the highest density clouds (Orion; dotted line in Figure 31) consistent with the idea that grain alignment is less efficient at higher densities.

These results are more difficult to interpret than for the polarization by ab- 
sorption case because the SMM flux is only a good tracer of dust column density if grains are either isothermal or warm enough so that the observations are made at wavelengths on the Rayleigh-Jeans tail. Neither of these conditions is necessarily true in molecular clouds.

Additionally, the polarization projected onto the plane of the sky allows only a lower limit to be placed on the grain alignment efficiency. While this is also true in the case of absorption, the projected magnetic field in molecular clouds can change more rapidly than in the diffuse ISM (i.e. on angular scales approaching the FIR/SMM resolution). An effort has been made to minimize this problem in Figure 3 by avoiding regions where the polarization position angle changes by more than 10 degrees between spatially adjacent points. Even with this position angle cut, the drop in polarization seen in Orion is partly due to magnetic field structure on scales below the $20^{\prime \prime}$ spatial resolution (Rao et al. 1998).

\section{Spectropolarimetry}

In both the cases of polarization by absorption and polarization by emission, multiwavelength polarimetry has been used to study magnetic fields and dust grain properties. However, it is in studies of the dust where spectropolarimetry is truly invaluable (see reviews by Martin 2006; Whittet 2003, 2004). We know that large grains (radii $\gtrsim 0.1 \mu \mathrm{m}$ ) are more efficient polarizers than small grains (radii $\lesssim$ $0.01 \mu \mathrm{m}$ ) because the polarization follows the extinction at infrared wavelengths, but not ultraviolet wavelengths. We know that silicate grains are better aligned than graphite grains because the spectral features of the O-Si bond are polarized, but carbon features are not. From the shape of the spectrum we know that the aligned grains are oblate (disc-like) rather than prolate (needle-like) (Draine \& Lee 1984; Lee \& Draine 1985; Hildebrand \& Dragovan 1995).

\subsection{Measured FIR/SMM Polarization Spectra}

Figure 4 shows two examples of multi-wavelength polarimetry of molecular clouds in the FIR/SMM. In comparing the percent polarization at different wavelengths one must consider that the changes can be due to changes in the strength or projection of the magnetic field, the efficiency of the alignment process, and the emissivity of the grains. All of these parameters may vary with position within the cloud. One may limit the effect of the magnetic field on these changes by avoiding regions where the position angle changes greatly from nearby points, or changes with wavelength. Such position angle changes can only be due to changes in the magnetic field.

Figure 5 5 shows polarization spectra in 6 molecular cloud envelopes. (Envelopes are defined as regions away from dense cores where opacity may affect the spectrum.) The spectrum falls from $60 \mu \mathrm{m}$ to $350 \mu \mathrm{m}$ and then rises from $350 \mu \mathrm{m}$ to $1300 \mu \mathrm{m}$. The uncertainties in these comparisons are large, and data exist both short- and long-ward of $350 \mu \mathrm{m}$ for only two of the clouds shown here (W51 and NGC2024). However, the trend is observed in multiple clouds, and in clouds where 

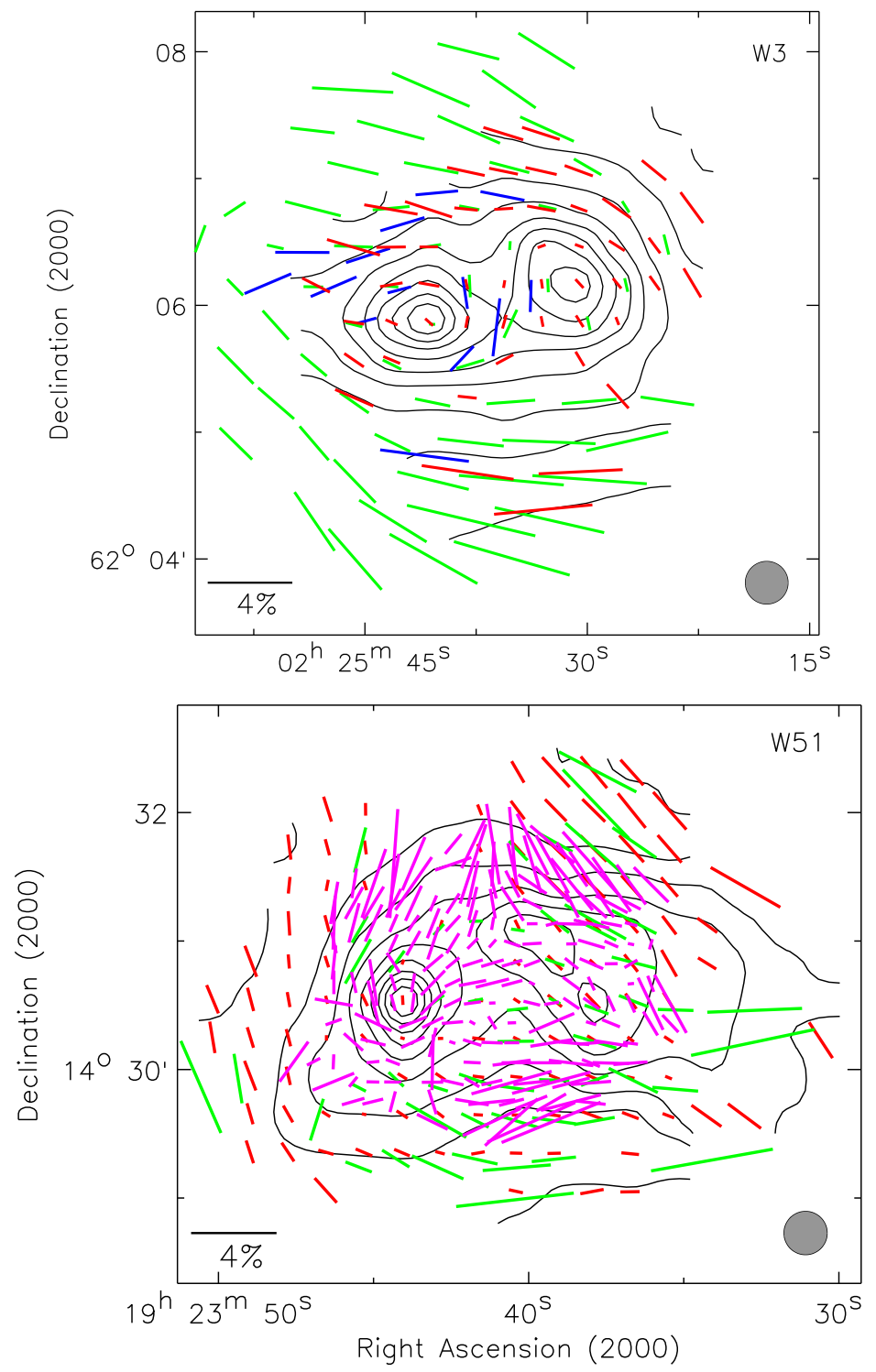

Fig. 4. Top: Polarization of W3 measured at $60 \mu \mathrm{m}$ (blue), $100 \mu \mathrm{m}$ (green), and $350 \mu \mathrm{m}$ (red). Notice the clockwise rotation of the vectors from 60 to 100 to $350 \mu \mathrm{m}$, signifying a change in the projected magnetic field along the line of sight, presumably due to differential rotation of the inner, warmer dust with respect to the outer, cooler dust (Schleuning et al. 2000). Bottom: Polarization of W51 at $100 \mu \mathrm{m}$ (green, Dotson et al. 2000), $350 \mu \mathrm{m}$ (red, Dotson et al. 2006), and $850 \mu \mathrm{m}$ (magenta, Chrvsostomou et al. 2002). Shaded circles show the $20^{\prime \prime}$ beam used to make the $350 \mu \mathrm{m}$ measurements and flux contours. 

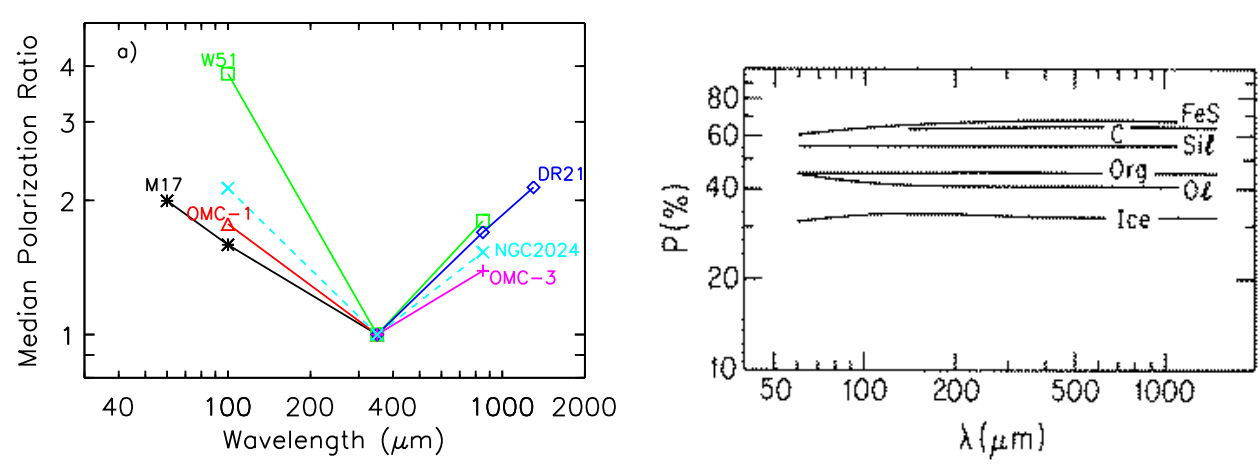

Fig. 5. Measured and predicted far-infrared/submillimeter polarization spectra for interstellar dust. a) Measured polarization spectra for molecular cloud envelopes, normalized at $350 \mu \mathrm{m}$ Vaillancourt 2002; Matthews et al. 2003; W51 $850 \mu \mathrm{m}$ data from Chrvsostomou et al. 2002). b) Estimated polarization spectra from individual grain species assuming perfect alignment, a single temperature, and a constant grain size and shape (Hildebrand et al. 1999).

data are available both short- and long-ward of the $350 \mu \mathrm{m}$ minimum. Continued observations of these and similar clouds at FIR/SMM wavelengths are needed to further confirm these results (Dowell et al. 2003; Novak et al. 2004; Li et al. 2006; Bastien. Jenness. \& Molnar 2005).

\subsection{Models of the Polarization Spectrum}

The wavelength dependence of polarization for specific types of grains can be calculated assuming uniform magnetic fields. Using the known dielectric properties of grain materials, Hildebrand et al. (1999) found that an ideal cloud composed of identical grains at a single temperature would produce a polarization nearly independent of wavelength for several different grain materials (Figure $5 \mathrm{~b}$ ).

Molecular clouds are certainly not ideal. This is confirmed by the polarization spectrum in Figure 5 a which clearly does not reproduce the prediction in Figure 5b. Real clouds are likely composed of multiple grain species with a distribution of temperatures. The total polarization is found by summing the polarized flux from each component (Hildebrand et al. 1999):

$$
P_{\mathrm{tot}}(\lambda)=\frac{1}{F_{\mathrm{tot}}(\lambda)} \sum_{i} p_{i} F_{i}(\lambda)
$$

where $F_{i}(\lambda)$ is the flux from species $i, F_{\text {tot }}(\lambda)=\sum_{i} F_{i}(\lambda)$, and $p_{i}$ is the wavelength independent polarization of species $i$ from Figure 5 b.

Consider a cloud composed of dust components at two temperatures, each with a different polarization efficiency. A wavelength dependent polarization spectrum 
then requires that two conditions be true:

$$
p_{1} \lessgtr p_{2} \quad \text { and } \quad \frac{1}{F_{1}} \frac{\mathrm{d} F_{1}}{\mathrm{~d} \lambda}>\frac{1}{F_{2}} \frac{\mathrm{d} F_{2}}{\mathrm{~d} \lambda}
$$

where the upper inequality holds for a falling spectrum $\left(\mathrm{d} P_{\text {tot }} / \mathrm{d} \lambda<0\right)$ and the lower inequality for a rising spectrum $\left(\mathrm{d} P_{\text {tot }} / \mathrm{d} \lambda>0\right)$. If the total emitted dust flux is modeled as an emissivity-modified blackbody with spectral index $\beta\left[F_{i}(\nu) \propto\right.$ $\left.\nu^{\beta_{i}} B_{\nu}\left(T_{i}\right)\right]$ then the second condition is equivalent to

$$
\begin{array}{lll}
T_{1}<T_{2} & \text { if } & \beta_{1}=\beta_{2} \\
\beta_{1}<\beta_{2} & \text { if } & T_{1}=T_{2} .
\end{array}
$$

The falling spectra between $60-350 \mu \mathrm{m}$ in Figure $5 \mathrm{~F}$ are consistent with a model in which the warmer grains are better aligned than the cold grains (Hildebrand et al. 1999; Vaillancourt 2002). Such regions may occur naturally in molecular clouds. Grains near embedded stars will be both warmed by stellar photons and be spun-up to suprathermal velocities by radiative torques, thereby increasing the grain alignment efficiency. Grains further from the star, or in optically thick clumps where stellar photons cannot penetrate, will be cooler and less affected by radiative torques. Vaillancourt (2002) has shown that the spectral energy distribution and polarization spectra in the Orion nebula (M42) H II region are consistent with this model.

The rising spectra longward of $350 \mu \mathrm{m}$ require another dust component with either a colder temperature or a lower spectral index than the dust in the dense clumps. Such dust may occur on the surface layers of molecular clouds where photons from the ISRF can impart radiative torques.

\section{Diffuse Clouds}

Diffuse low density clouds present a simpler case for studies of interstellar dust grains than molecular clouds. In clouds transparent to starlight all grains are exposed to the same, nearly isotropic, radiation source. Such clouds would represent the ideal case of Figure 5 except that grain species with different emissivities will have different equilibrium temperatures. The diffuse infrared cirrus clouds observed at high Galactic latitude by the Infrared Astronomical Satellite (IRAS; Low et al. 1984) are the best observational examples of ideal clouds. These clouds are distributed over most of the sky and are bright enough (10-100 MJy/sr at $\lambda=100-200 \mu \mathrm{m})$ to be detectable with the next generation of airborne photometers (Harper et al. 2000) and polarimeters (Dowell et al. 2003).

The FIR/SMM spectral energy distribution of the Galaxy can be modeled by two dust components with mean temperatures of $9.4 \mathrm{~K}$ and $16 \mathrm{~K}$ and spectral indices of 1.7 and 2.7, respectively (Finkbeiner. Davis. \& Schlegel 1999). Measurements of the optical properties of graphites and silicates (Draine \& Lee 1984; Pollack et al. 1994) led Finkbeiner et al. (1999) to tentatively identify silicate grains with the $\beta=1.7$ component and carbonaceous grains with the $\beta=2.7$ 
component. Silicate grains reach colder equilibrium temperatures than graphites when both are exposed to the ISRF. (Draine \& Lee estimated higher temperatures than those of Finkbeiner et al.) With silicate colder than graphite, and silicate grains more polarized than carbon grains (Whittet 2004) equations (4.2)-(4.4) predict that polarized emission from diffuse cirrus clouds will increase with wavelength (6.3 Hildebrand \& Kirbv 2004). Tests of this prediction require observations at FIR/SMM wavelengths. Polarimetric observations from the Stratospheric Observatory for Infrared Astronomy (SOFIA) will cover the $60-215 \mu \mathrm{m}$ range (Dowell et al. 2003). Submillimeter wavelengths can be covered by Planck at 350 - $1400 \mu \mathrm{m}$ (Tauber 2004), Archeops at $850 \mu \mathrm{m}$ (Ponthieu 2006: Benoit et al. 2004), and $B L A S T$ at $250-500 \mu \mathrm{m}$ (Netterfield 2006).

\section{Microwave Emission}

\subsection{Total Microwave Flux Spectra}

Several studies covering large areas on the sky in the $10-90 \mathrm{GHz}$ range have reported microwave emission which is highly correlated with thermal dust emission (Kogut et al. 1996; de Oliveira-Costa et al. 1997; Leitch et al. 1997). The microwave flux is larger than that expected from a combination of only thermal dust, free-free emission, and synchrotron emission (de Oliveira-Costa et al. 1999; Draine \& Lazarian 1998a), suggesting the existence of a new emission component in this spectral region. The strong correlations between this excess microwave flux and dust emission at shorter far-infrared wavelengths have led to two models in which dust is the source of the excess flux (see review by Lazarian \& Finkbeiner 2003).

In the first model, small grains (radii $\lesssim 0.001 \mu \mathrm{m}$ ) have dipole moments, spin rapidly, and emit electric dipole radiation at microwave frequencies (Draine \& Lazarian 1998a b). These so-called "spinning-dust" grains are the same small grains responsible for extinction at ultraviolet wavelengths in diffuse regions of the ISM. In the second mechanism, thermal fluctuations in large grains (radii $\gtrsim 0.1 \mu \mathrm{m}$ ), the same grains responsible for emission in the far-infrared, lead to fluctuations of the grains' magnetization. The result is magnetic dipole emission from "vibratingdust" grains at microwave frequencies (Draine \& Lazarian 1999).

Figure 6] shows three examples of observations in which the microwave emission cannot be modeled by only free-free, synchrotron, and thermal dust emission. The excess fluxes are well fitted to electric dipole emission from spinning dust. However, the model uncertainties are large enough that the flux data are not sufficient to rule out vibrating magnetic dust as a significant component (Hildebrand \& Kirbv 2004; Finkbeiner, Langston, \& Minter 2004).

Regardless of the actual carrier of the microwave excess, it is a large contributor to the total flux at $5-50 \mathrm{GHz}$ and may have a significant impact on measurements of the cosmic microwave background. Models consisting of only synchrotron or free-free emission below $100 \mathrm{GHz}$ can be ruled out for the regions shown in Figure 6. The rising spectra in the $5-20 \mathrm{GHz}$ range are inconsistent with the expected 


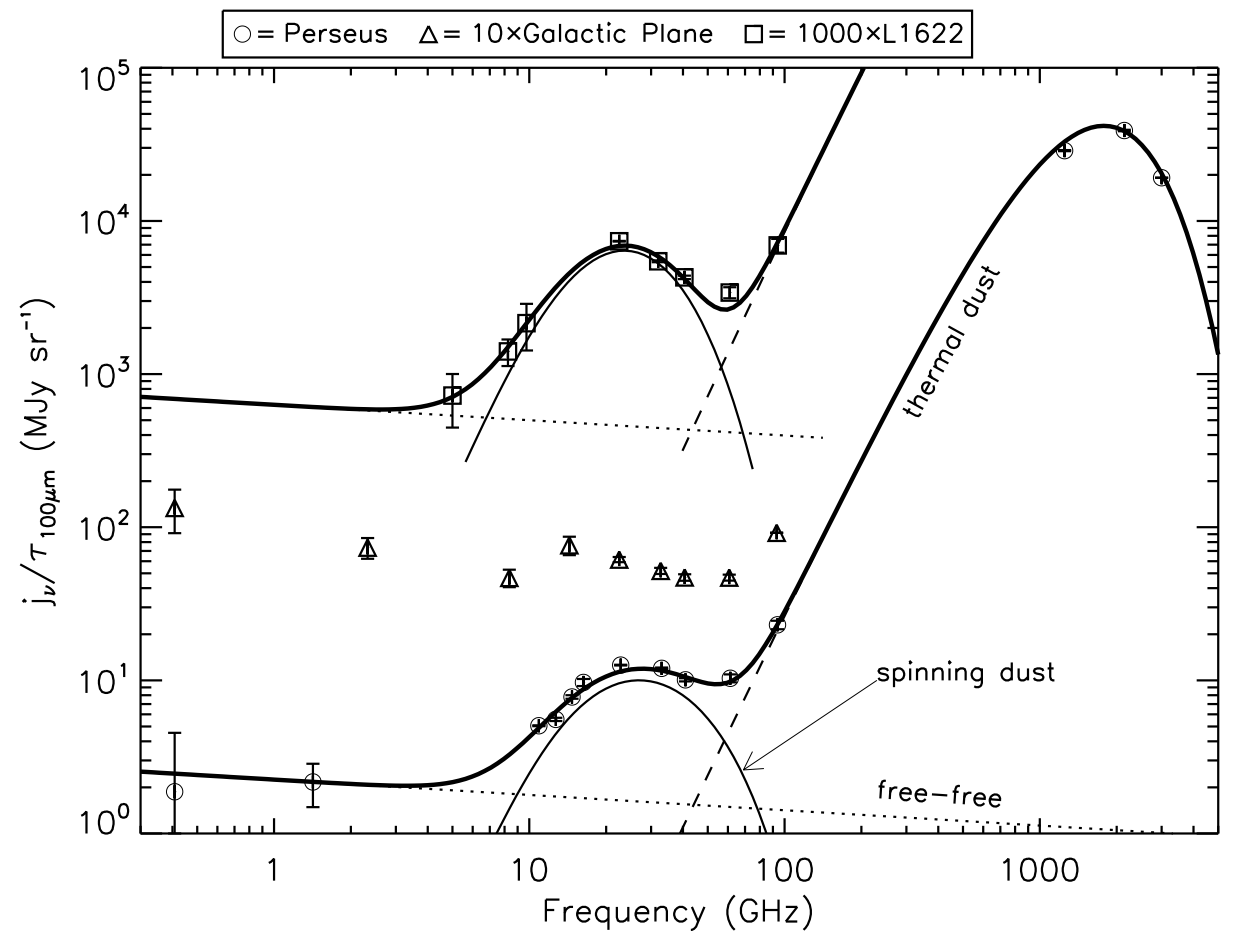

Fig. 6. Three examples of spectral energy distributions with excess microwave emission. The flux is in units of emission per $100 \mu \mathrm{m}$ optical depth (e.g. Finkbeiner 2004). Circles: G159.6-18.5 in the Perseus molecular cloud (Watson et al. 2005). Triangles: a region of diffuse emission in the Galactic plane $\left(|b|<4^{\circ}, l \sim 45^{\circ}\right), \times 10$ Finkbeiner. Langston. \& Minter 2004). Squares: the dark cloud Lynds $1622, \times 1000$ (Finkbeiner 2004; Hildebrand \& Kirbv 2004). Also shown are possible total emission models for the Perseus and Lynds 1622 clouds (thick solid lines). Both models include thermal dust emission (dashed), free-free emission (dotted), and spinning dust emission (thin solid lines). The Galactic plane emission can also be modeled with a combination of these mechanisms and synchrotron emission (Finkbeiner et al. 2004).

spectral shape of these two mechanisms.

\subsection{Microwave Polarization Spectra}

Extension of the polarization spectrum to microwave wavelengths is a matter of adding the polarized contributions from free-free, synchrotron, and spinning and/or vibrating dust to equation (4.1). One can expect that the small grains responsible for electric dipole emission will be poorly aligned with magnetic fields (Martin 2006; Whittet 2004). Consequently, they will emit very little polarized radiation. Lazarian \& Draine (2000) provide an upper limit on the alignment of 
spinning dust grains. They predict spinning dust will be polarized no more than $7 \%$ at $2 \mathrm{GHz}$, dropping to $<0.5 \%$ at frequencies above $30 \mathrm{GHz}$. The polarization position angle for spinning grains will be perpendicular to the aligning field, parallel to the polarized emission from thermal dust.

However, magnetic dipole emission from vibrating grains should be highly polarized. Draine \& Lazarian (1999) predict a polarization level as high as $40 \%$ for grains with single magnetic domains. Additionally, for certain grain shapes, the polarization position angle may flip by 90 degrees as the frequency changes. At high frequencies the polarization will be perpendicular to the magnetic field, as it is for the cases of spinning and thermal dust. At low frequencies the polarization will be parallel to the field.

The different polarization spectra for spinning and vibrating dust should allow for observational tests of these mechanisms where the flux data alone did not. The flip in the polarization position angle with frequency is the definitive signature of magnetic dipole emission. With the exception of frequencies near the position angle flip, magnetic dipole radiation from all single-domain grains is highly polarized, whereas emission from spinning grains is mostly unpolarized above $30 \mathrm{GHz}$ $(\lambda<10 \mathrm{~mm})$.

\subsection{Flux and Polarization Spectra of Diffuse Clouds}

Consider a diffuse cloud composed of thermal dust at two temperatures ( 9 and $16 \mathrm{~K})$, spinning dust ${ }^{1}$, and free electrons. The emission spectrum of such a cloud will be dominated by thermal dust at $\lambda \sim 50 \mu \mathrm{m}$ to a few millimeters $(\nu \sim 100-$ $6000 \mathrm{GHz}$ ). At longer wavelengths it may be dominated by electric dipole emission from spinning dust, and at yet longer wavelengths by free-free and/or synchrotron emission. Figure 7 shows a schematic spectrum of a hypothetical cloud where synchrotron is subdominant in the entire $50 \mu \mathrm{m}-5 \mathrm{~cm}$ range.

Figure 7 also shows a predicted polarization spectrum for such a cloud. If we assume that the warm thermal dust component is unpolarized while the cold component is polarized $(P \sim 10 \%$ in Figure 7$)$ then the polarization spectrum will rise in the FIR/SMM (15). The polarization drops at $\lambda>1 \mathrm{~mm}(\nu<300 \mathrm{GHz})$ where the flux is dominated by unpolarized radiation from spinning dust and free-free emission. The polarization may rise for $\lambda>1 \mathrm{~cm}(\nu<30 \mathrm{GHz})$ if the prediction for the polarization of spinning dust is correct (Lazarian \& Draine 2000).

If the dominant source of excess microwave emission is magnetic dipole emission from vibrating dust then the polarization spectrum in Figure [7 may rise at $\lambda>$ $1 \mathrm{~mm}$ where this emission dominates that from thermal dust. In this toy model, the polarization from these two different mechanisms differs by $\Delta P \sim 20 \%$ at $40 \mathrm{GHz}$, well within the uncertainties of the current generation of polarimeters $(\Delta P \lesssim 1 \%)$. Future polarization data from WMAP (Hinshaw et al. 2003) and Planck (Tauber 2004) should clearly distinguish between spinning and vibrating dust.

\footnotetext{
${ }^{1}$ Spinning dust predictions were downloaded from/http://www.astro.princeton.edu/ draine/dust/dust.html
} 


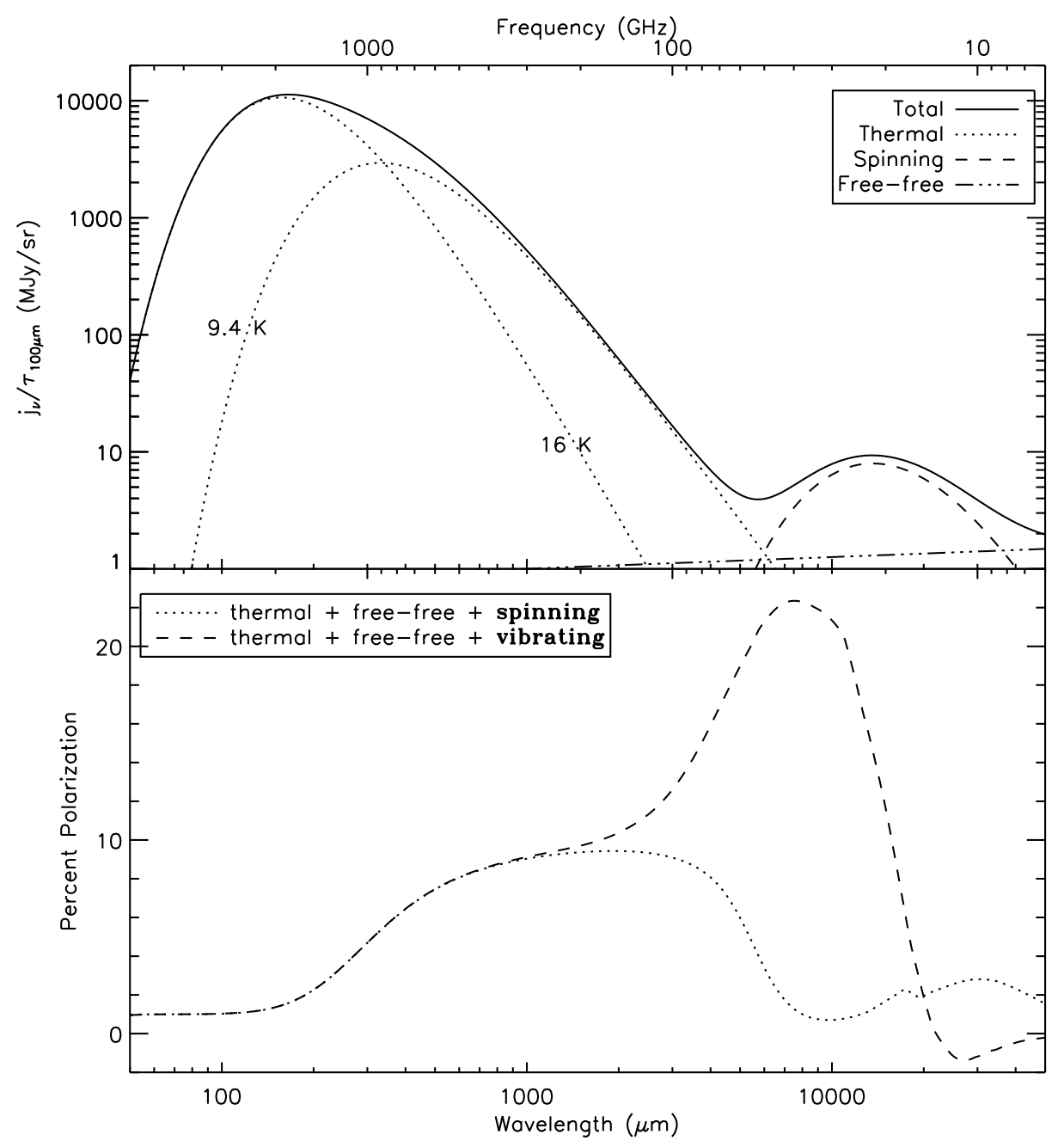

Fig. 7. Top: Far-infrared - microwave spectral energy distribution of a hypothetical diffuse cloud. Flux components include thermal emission from dust at $9.4 \mathrm{~K}$ and $16 \mathrm{~K}$ (Finkbeiner et al. 1999), electric dipole emission from spinning dust (cold neutral medium prediction of Draine \& Lazarian 1998b), and free-free emission. Bottom: Predicted polarization spectra for emission including upper limits for spinning dust and vibrating magnetic dust. Thermal dust is assumed polarized at the $10 \%$ level for the $9.4 \mathrm{~K}$ component and unpolarized for the $16 \mathrm{~K}$ component. Spinning dust polarization is taken from the resonance relaxation model of Lazarian \& Draine (2000). The vibrating dust SED and polarization curves are for the Fe-rich material X4 of Draine \& Lazarian (1999). In order to calculate the polarized flux from magnetic dust the SED peak of magnetic dust (not shown in top panel) is scaled to match the peak of the spinning dust SED. The polarization is for single-domain grains with axes ratios 1:1.5:2 (Draine \& Lazarian 1999). Free-free emission is assumed to be unpolarized. 


\section{Summary}

Dust grains aligned with magnetic fields in the interstellar medium are responsible for both polarization by absorption at optical wavelengths and polarization by emission at far-infrared and submillimeter wavelengths. Observations of the absorption and emission trace different regions of the ISM but both are consistent with the existence of radiative torques which spin grains up to the suprathermal angular velocities needed for magnetic alignment.

In molecular clouds the FIR/SMM polarization is strongly dependent on wavelength. We have attributed this wavelength dependence to sampling different grain

populations at different temperatures. This wavelength dependence provides an opportunity to study, in different physical environments, the grain properties, the alignment mechanism and its efficiency, and magnetic fields. To date, most observations of polarized emission have been in the densest regions of the ISM. Extending these observations to regions of the diffuse ISM will provide additional tests of grain and alignment models. Instruments on board the Stratospheric Observatory for Infrared Astronomy (SOFIA) will have the sensitivity to detect the total (HAWC: Harper et al. 2000) and polarized (Hale: Dowell et al. 2003) flux in diffuse infrared cirrus clouds. Instruments, including Planck and WMAP, designed to separate polarized Galactic foregrounds from the CMB will be able to extend the diffuse cloud polarization spectrum to longer SMM and microwave wavelengths.

The polarization state of the excess microwave emission observed at frequencies of $10-90 \mathrm{GHz}$ is key to an accurate measurement of polarization from the cosmic microwave background. Total power observations are currently unable to conclusively rule out either of the two leading candidates for this emission: electric dipole emission from small spinning grains and magnetic dipole emission from larger vibrating grains. Polarization observations made with WMAP and Planck will place limits on the emission contributions from these two sources.

I would like to thank Roger Hildebrand, Alex Lazarian, and Darren Dowell for valuable comments on drafts of this manuscript. This work has been supported in part by NSF grants AST-0204886 and AST-0505124.

\section{References}

Aitken, D. K. 1996, in ASP Conf. Ser. 97, Polarimetry of the Interstellar Medium, ed. W. G. Roberge \& D. C. B. Whittet, 225

Arce, H. G., Goodman, A. A., Bastien, P., Manset, N., \& Sumner, M. 1998, ApJ, 499, L93

Bastien, P. 2006, this volume

Bastien, P., Jenness, T., \& Molnar, J. 2005, in ASP Conf. Ser. 343, Astronomical Polarimetry - Current Status and Future Directions, ed. A. Adamson, in press 
Benoit, A., et al. 2004, A\&A, 424, 571

Berdyugin, A., Piirola, V., \& Teerikorpi, P. 2004, A\&A, 424, 873

Cho, J. \& Lazarian, A. 2005, ApJ, 631, 361

Chrysostomou, A., Aitken, D. K., Jenness, T., Davis, C. J., Hough, J. H., Curran, R., \& Tamura, M. 2002, A\&A, 385, 1014

Chuss, D. T., Davidson, J. A., Dotson, J. L., Dowell, C. D., Hildebrand, R. H., Novak, G., \& Vaillancourt, J. E. 2003, ApJ, 599, 1116

Crutcher, R. M. 2006, this volume

Davis, L. J. \& Greenstein, J. L. 1951, ApJ, 114, 206

de Oliveira-Costa, A., Kogut, A., Devlin, M. J., Netterfield, C. B., Page, L. A., \& Wollack, E. J. 1997, ApJ, 482, L17

de Oliveira-Costa, A., Tegmark, M., Gutierrez, C. M., Jones, A. W., Davies, R. D., Lasenby, A. N., Rebolo, R., \& Watson, R. A. 1999, ApJ, 527, L9

Dolginov, A. Z. 1972, Ap\&SS, 18, 337

Dolginov, A. Z. \& Mytrophanov, I. G. 1976, Ap\&SS, 43, 257

Dotson, J. L., Davidson, J., Dowell, C. D., Schleuning, D. A., \& Hildebrand, R. H. 2000, ApJS, 128, 335

Dotson, J. L., Dowell, C. D., Hildebrand, R. H., Kirby, L., \& Vaillancourt, J. E. 2006, in preparation

Dowell, C. D., Davidson, J. A., Dotson, J. L., Hildebrand, R. H., Novak, G., Rennick, T. S., \& Vaillancourt, J. E. 2003, in Proc. SPIE 4843, Polarimetry in Astronomy, ed. S. Fineschi, 250

Draine, B. T. \& Lazarian, A. 1998a, ApJ, 494, L19

—. 1998b, ApJ, 508, 157

—. 1999, ApJ, 512, 740

Draine, B. T. \& Lee, H. M. 1984, ApJ, 285, 89, erratum: 1987 ApJ, 318, 485

Draine, B. T. \& Weingartner, J. C. 1996, ApJ, 470, 551

—. 1997, ApJ, 480, 633

Finkbeiner, D. P. 2004, ApJ, 614, 186

Finkbeiner, D. P., Davis, M., \& Schlegel, D. J. 1999, ApJ, 524, 867

Finkbeiner, D. P., Langston, G. I., \& Minter, A. H. 2004, ApJ, 617, 350 
Gold, T. 1952, MNRAS, 112, 215

Goodman, A. A., Jones, T. J., Lada, E. A., \& Myers, P. C. 1995, ApJ, 448, 748

Harper, D. A., et al. 2000, in Proc. SPIE 4014, Airborne Telescope Systems, ed. R. K. Melugin \& H.-P. Roeser, 43

Heiles, C. 2000, AJ, 119, 923

Hildebrand, R. \& Kirby, L. 2004, in ASP Conf. Ser. 309, Astrophysics of Dust, ed. A. N. Witt, G. C. Clayton, \& B. T. Draine, 515

Hildebrand, R. H. 1983, QJRAS, 24, 267

—. 1988, QJRAS, 29, 327

Hildebrand, R. H. 2001, in Astrophysical Spectropolarimetry, ed. J. TrujilloBueno, F. Moreno-Insertis, \& F. Sanchez (Cambridge: Cambridge University Press), 265

Hildebrand, R. H., Dotson, J. L., Dowell, C. D., Schleuning, D. A., \& Vaillancourt, J. E. 1999, ApJ, 516, 834

Hildebrand, R. H. \& Dragovan, M. 1995, ApJ, 450, 663

Hinshaw, G., et al. 2003, ApJS, 148, 63

Houde, M., Dowell, C. D., Hildebrand, R. H., Dotson, J. L., Vaillancourt, J. E., Phillips, T. G., Peng, R., \& Bastien, P. 2004, ApJ, 604, 717

Kogut, A., Banday, A. J., Bennett, C. L., Gorski, K. M., Hinshaw, G., \& Reach, W. T. 1996, ApJ, 460, 1

Lazarian, A. 2003, Journal of Quantitative Spectroscopy and Radiative Transfer, 79,881

Lazarian, A. \& Cho, J. 2005, in ASP Conf. Ser. 343, Astronomical Polarimetry Current Status and Future Directions, ed. A. Adamson, in press

Lazarian, A. \& Draine, B. T. 1997, ApJ, 487, 248

—. 1999a, ApJ, 520, L67

—. 1999b, ApJ, 516, L37

—. 2000, ApJ, 536, L15

Lazarian, A. \& Finkbeiner, D. 2003, New Astronomy Review, 47, 1107

Lazarian, A., Goodman, A. A., \& Myers, P. C. 1997, ApJ, 490, 273

Lazarian, A. \& Yan, H. 2004, in ASP Conf. Ser. 309: Astrophysics of Dust, ed. A. N. Witt, G. C. Clayton, \& B. T. Draine, 479 
Lee, H. M. \& Draine, B. T. 1985, ApJ, 290, 211

Leitch, E. M., Readhead, A. C. S., Pearson, T. J., \& Myers, S. T. 1997, ApJ, 486, L23

Li, H., et al. 2006, in Proc. SPIE, Astronomical Telescopes and Instrumentation, Orlando, FL, 24-31 May 2006, in prep.

Low, F. J., et al. 1984, ApJ, 278, L19

Martin, P. G. 2006, this volume, astro-ph/0606430

Matthews, B. C., Chuss, D., Dotson, J., Dowell, D., Hildebrand, R., Johnstone, D., \& Vaillancourt, J. 2003, in Chemistry as a Diagnostic of Star Formation, ed. C. L. Curry \& M. Fich, 145

Matthews, B. C., Wilson, C. D., \& Fiege, J. D. 2001, ApJ, 562, 400

Netterfield, B. 2006, this volume

Novak, G., et al. 2004, in Proc. SPIE 5498, Millimeter and Submillimeter Detectors for Astronomy II, ed. J. Zmuidzinas, W. S. Holland, \& S. Withington, 278

Pollack, J. B., Hollenbach, D., Beckwith, S., Simonelli, D. P., Roush, T., \& Fong, W. 1994, ApJ, 421, 615

Ponthieu, N. 2006, this volume

Purcell, E. M. 1979, ApJ, 231, 404

Rao, R., Crutcher, R. M., Plambeck, R. L., \& Wright, M. C. H. 1998, ApJ, 502, L75

Roberge, W. G. 2004, in ASP Conf. Ser. 309, Astrophysics of Dust, ed. A. N. Witt, G. C. Clayton, \& B. T. Draine, 467

Schleuning, D. A., Vaillancourt, J. E., Hildebrand, R. H., Dowell, C. D., Novak, G., Dotson, J. L., \& Davidson, J. A. 2000, ApJ, 535, 913

Tauber, J. A. 2004, in The Magnetized Interstellar Medium, ed. B. Uyaniker, W. Reich, \& R. Wielebinski, 191

Vaillancourt, J. E. 2002, ApJS, 142, 53

Watson, R. A., Rebolo, R., Rubiño-Martín, J. A., Hildebrandt, S., Gutiérrez, C. M., Fernández-Cerezo, S., Hoyland, R. J., \& Battistelli, E. S. 2005, ApJ, 624, L89

Whittet, D. C. B. 2003, Dust in the Galactic Environment, 2nd edn., Series on Astronomy and Astrophysics (Philadelphia: Institute of Physics Publishing) 
Whittet, D. C. B. 2004, in ASP Conf. Ser. 309, Astrophysics of Dust, ed. A. N. Witt, G. C. Clayton, \& B. T. Draine, 65

Whittet, D. C. B., Gerakines, P. A., Hough, J. H., \& Shenoy, S. S. 2001, ApJ, 547,872 